\title{
Epicondylitis Band or Corticosteroid Injection for Lateral Epicondylitis Treatment?
}

\author{
Ertuğrul Allahverdi ${ }^{1}$, Tülay Diken Allahverdi ${ }^{2}$, Uğur Türktaş ${ }^{3}$ \\ ${ }^{1}$ Kafkas University Faculty of Medicine Department of Orthoppaedics and Traumatology Kars/Turkey \\ ${ }^{2}$ Kafkas University Faculty of Medicine Department of General Surgery, Kars/Turkey \\ 3 Turgut Özal University Faculty of Medicine Department of Orthoppaedics and Traumatology_Malatya/Turkey
}

Received: 26 November 2015 accepted: 06 April 2016/ published online 27 April 2016

(C) Ordu University Institute of Health Sciences, Turkey, 2016

\begin{abstract}
Objective: Lateral epicondylitis has been identified as tendinosis developing in the region where extensor muscles. Conservative methods are first used in the treatment of acute lateral epicondylitis. One of the most widely used methods is corticosteroid and local anesthetic injection. Injection treatment is preferred because good results can be obtained in a short time. Comparison of the short- and medium-term results of the epicondylitis band and corticosteroid and local anesthetic injections for lateral epicondylitis. Case Series. This was a retrospective and comparative study related to acute lateral epicondylitis treatment.

Methods: The patient groups named A and B received two different treatments. The information obtained from the charts of 356 patients who were referred to the orthopedics departments of two separate hospitals between February 2010 and June 2013. There were 151 patients in Group A and 205 patients in Group B. Group A received the epicondylitis band and Group B corticosteroid and local anesthetic injections. Both groups were also given stretch exercises and nonsteroidal anti-inflammatory drugs. Patients were checked at week 1, 4, 12, and 24 and the Quick DASH scoring was used on week 12 and 24.

Results: On the 3rd month of treatment, Group A showed recovery rates of moderate, good and full in $18.5 \%$, $32.5 \%$ and $30.5 \%$ respectively while these rates were $33.2 \%, 13.7 \%$ and $25.4 \%$ in Group B. The same rates at the end of month 6 were $17.2 \%, 41.1 \%$ and $17.2 \%$ in Group A and $28.3 \%, 1 \%$ and $17.2 \%$ in Group B. We see that treatment of group A was more successful than group B in the 3rd month $(p<0,05)$, and much more successful than group B in the 6th month $(p<0,001)$ in terms of the number of patients who were successfully and unsuccessfully treated according to the Quick DASH scores.

Conclusions: Combined treatment (physical therapy, nonsteroidal anti-inflammatory drugs) with an epicondylitis band was more effective than combined treatment with steroid and local anesthetic injections in acute lateral epicondylitis.

Key words: Acute Lateral Epicondylitis, Epicondylopathy, Steroids, Quick DASH Score, Epicondylitis Band

Address for correspondence/reprints:

Tülay Diken Allahverdi

Telephone number: +90 5066319651

E-mail: drtulaydiken@hotmail.com

DOI: 10.19127/mbsjohs.35948

\section{Introduction}

Lateral epicondylitis has been identified as tendinosis developing in the region where extensor muscles and especially the extensor carpi radialisbrevis muscle originate after these muscles are forced or undergo repetitive stress at the lateral humeral epicondyle and the condition can also be
\end{abstract}


accompanied by microtrauma or partial tears that can progress to complete tears (Nirschl et al., 1979). It can be easily diagnosed with a good medical history from the patient and physical examination and it is typically characterized by recurrence of the pain after repeated excessive and forced movements in the arm. The value of radiographs in the diagnosis is very low (Pomerance et al., 2002). The presence of pain in the lateral condyle with repeated supination and pronation while the elbow is in extension or the wrist is forced to extension against resistance is enough to diagnose lateral epicondylitis (Nirschl et al., 1979; Jobe et al., 1994; Cohen et al., 2008).

Lateral epicondylitis was first identified in 1873 by Runge as 'handwriting cramp' (Lopes-Martins et al., 2006). The mean incidence is $1-3 \%$ (Smidt et al., 2002). It is most commonly seen in 4th and 5th decades (Lopes-Martins et al., 2006). The disorder is 7-10 times more common than medial epicondylitis (Ciccotti et al., 2004). It improves in 1 year (6-24 months) on average with treatment (Smidt et al., 2002). The first option is generally conservative treatment (Papa et al., 2012). The conservative treatment methods used are usually local corticosteroids + local anesthetic injection, epicondylitis band application, extracorporeal shock wave therapy (ESWT), acupuncture, physical therapy, ultrasound phonophoresis, electrotherapy and NSA iontophoresis treatments, transcutaneous electrical nerve stimulation (TENS), low-grade laser treatment, autologous blood injection, topical nitrates, and type A Botulinum Toxin (Botox) injection (Smidt et al., 2002; Johns et al., 2002; Uzunca et al., 2007; Chesterton et al., 2009; Papa et al., 2012). Surgical procedures can be considered in cases that do not respond to conservative treatment for 6-9 months. Many surgical techniques have been described (Nirschl et al., 1979).

The epicondylitis band is thought to have an effect by decreasing the load at the initial adhesion sites of the extensor muscles and there are various studies on the mechanism of action (Meyer et al., 2002; Altan et al., 2008). Corticosteroid + local anesthetic drug injections show their effect in the same area with their anti-inflammatory feature (Meyer et al., 2002; Struijs et al., 2004).

Lateral epicondylitis pain can be explained by the tenopathy and arthrogenic and neurogenic mechanisms. Compression of the radial nerve is effective in the neurogenic mechanism. Erak et al (2004) identified that the deep branch of the radial nerve started the pain with the increase of the tensile forces of the extensors on the lateral epicondyle in a biomechanical study. Decompression of the radial nerve should be among the alternatives when considering a surgical procedure in resistant cases (Meyer et al., 2002).

\section{Materials and Methods}

Our retrospective, comparative study using the chart review model was conducted via the information obtained from the charts of 356 patients who were referred to the orthopedics departments of two separate hospitals from the outpatient departments of various specialties between February 2010 and June 2013 with symptoms of elbow pain and limitation of movement and diagnosed with unilateral lateral epicondylitis with orthopedic clinical examinations and investigations, whose initial Quick Dash scores were similar, who had difficulty in performing their daily activities, and who were followed-up and treated. The distribution of the patients to the two orthopedics outpatient departments was realized directly according to the patients' presentations. The approval of the local ethics committee was obtained and the age, gender, occupation, treatment choice, results and Quick DASH scores were recorded from the files of the patients included in the study. The patients were distributed into two separate treatment groups as A and B. There were 151 patients in group A and 205 patients in group B. Patients who had received another epicondylitis treatment within the last 6 months, diagnosed with bilateral epicondylitis, had cervical radiculopathy or systemic musculoskeletal and neurological disorders, had a history of surgical intervention or trauma to the elbow, had chronic diseases, patients with contraindications for corticosteroids, pregnant or nursing women, patients younger than 16 years, those receiving oral or systemic steroid therapy, and patients with psychiatric problems were excluded from the study.

Group A received an epicondylitis band + combined treatment to the forearm 3-4 cm distal from the lateral epicondyle (Figure 1 ) for 3 months and group B received corticosteroids and local anesthetic injection (betamethasone $2 \mathrm{mg} 1$ $\mathrm{ml}$ and $2 \%$ lidocaine $1 \mathrm{ml}$ ) (Figure 2 ) + combined treatment. An injection was administered one more time 4 weeks later to some of the patients in Group $\mathrm{B}$ according to the treatment response. 
The combined treatment protocol for both groups included concentric contractions, eccentric exercises (Figure 3 and 4), NSAIDs (oral/topical), and cold/hot compress applications. The follow-up examinations were performed at the 1st, 4th, 12th and 24th weeks. The results obtained during the follow-ups at the 12th and 24th weeks were evaluated with Quick DASH Scoring (Öksüz et al., 2006; Franchignoni et al., 2010). Quick DASH scores were defined as $0 \%$ none, $25 \%$ partial, $50 \%$ moderate, $75 \%$ good, and $100 \%$ full improvement.

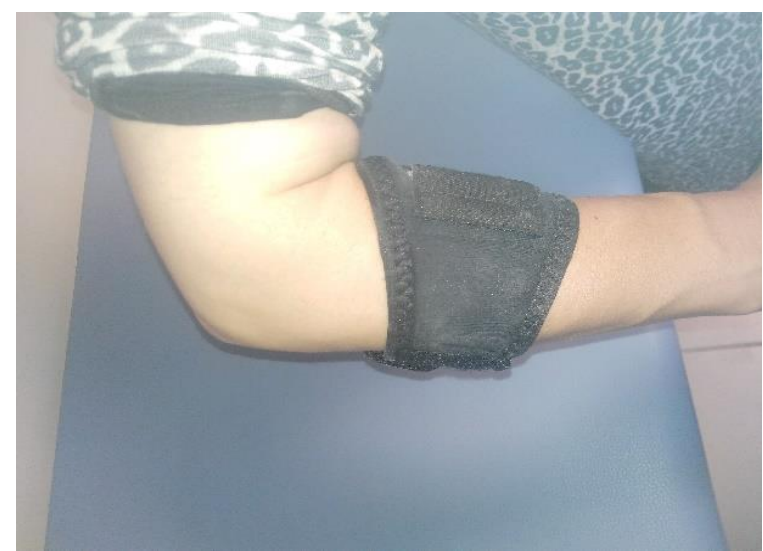

Figure 1: Therapeutic forearm band for tennis elbow (lateral epicondylitis)

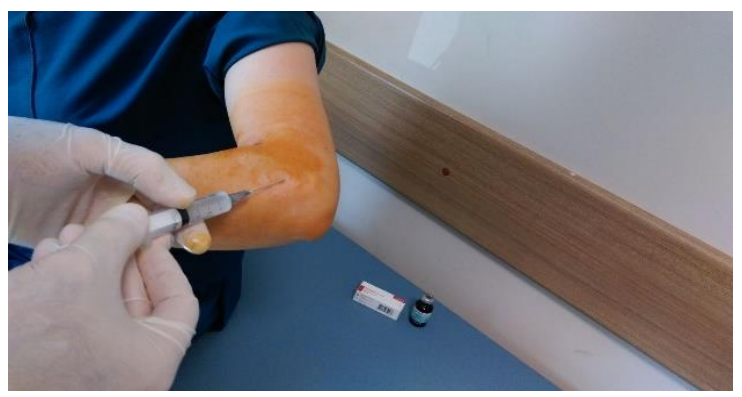

Figure 2: Cortcosteroid with local anesthetic injections for treatment of lateral epicondylitis of elbow

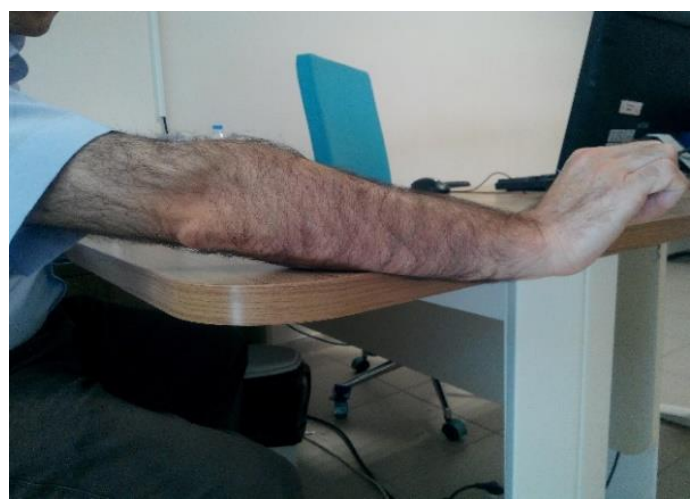

Figure 3: Eccentric contraction exercise

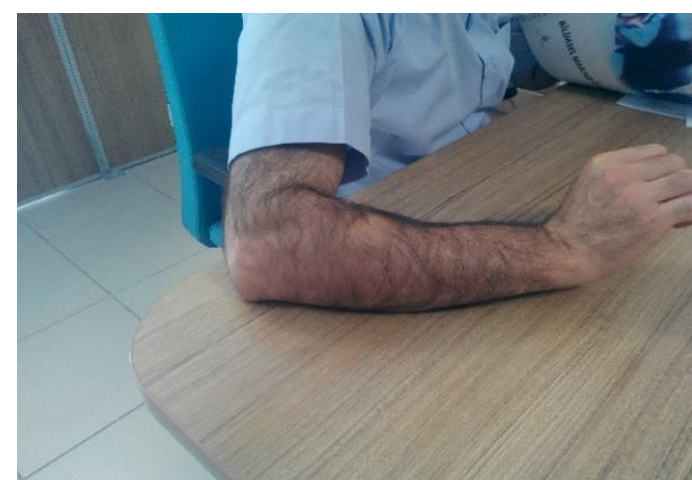

Figure 4: Concentric contraction exercise

Statistics: Statistical analyses were performed with the SPSS 13 program using the $\mathrm{X}^{2}$ test and comparison of percentages.

\section{Results}

A total of 356 lateral epicondylitis patients consisting of $231(64.8 \%)$ females and 125 $(35.2 \%)$ males were included in 2 groups in our study. The mean age of the patients was $48.5+/-$ 10.7 years. The affected side was the right in 210 (58.9\%) patients and the left in $146(41.1 \%)$ patients. The patients were diagnosed with physical examination. The mean duration of presentation was 1.9 weeks in group A and 1.7 weeks in group B. The patients had usually presented shortly after the beginning of the symptoms. Conservative treatment (oral/topical NSAID, epicondylitis band, oral paracetamol) had previously been used by $80(22.4 \%)$ patients. We evaluated 151 patients in group A and 205 patients in group B. A surgical procedure was performed in $6(3.9 \%)$ patients in group A and $18(8.7 \%)$ patients in group B as there was no decrease in symptoms at the end of the 6-month treatment period. A second injection was administered to 54 $(26.3 \%)$ patients in group B at the 4th week. Group A patients fully complied with the epicondylitis band application. Age, gender are presented in Table-1 by group.

The patients were evaluated with specific Quick DASH scoring in the 12th and 24th weeks in our study. Quick DASH scores according to the groups are presented in Graphic 1. We accepted moderate, good and very good Quick DASH scores as successful treatment and no benefit and little benefit results as unsuccessful treatment.

According to these results, the short-term improvement after 3 months of treatment was moderate in $18.5 \%$, good in $32.5 \%$ and complete in $30.5 \%$ in group A and the treatment was successful in $81.5 \%$ and unsuccessful in $18.5 \%$. 
Table 1: According to the results of the treatment of group A and B success graphic

\begin{tabular}{|c|c|c|c|c|c|c|c|c|c|c|c|c|}
\hline \multirow{3}{*}{ Patient groups } & \multicolumn{4}{|c|}{ Gender } & \multicolumn{8}{|c|}{ Age (Years) } \\
\hline & \multicolumn{2}{|c|}{ Famale } & \multicolumn{2}{|c|}{ Male } & \multicolumn{2}{|l|}{$15-20$} & \multicolumn{2}{|c|}{$20-40$} & \multicolumn{2}{|l|}{$40-60$} & \multicolumn{2}{|c|}{$60-85$} \\
\hline & $\mathbf{n}$ & $\%$ & $\mathbf{n}$ & $\%$ & $\mathbf{n}$ & $\%$ & $\mathbf{n}$ & $\%$ & $\mathbf{n}$ & $\%$ & $\mathbf{n}$ & $\%$ \\
\hline Group A & 102 & 67,54 & 49 & 32,45 & 3 & 1,98 & 30 & 19,86 & 106 & 70,19 & 12 & 7,94 \\
\hline Group B & 129 & 62,92 & 76 & 37,07 & 2 & 0,97 & 27 & 13,17 & 139 & 67,8 & 37 & 18,04 \\
\hline \multirow{3}{*}{ Patient groups } & \multicolumn{4}{|c|}{ Affected side } & \multicolumn{8}{|c|}{ Occupation } \\
\hline & \multicolumn{2}{|c|}{ Right } & \multicolumn{2}{|c|}{ Left } & \multicolumn{2}{|c|}{ Housewife } & \multicolumn{2}{|c|}{ Worker } & \multicolumn{2}{|c|}{ Officer } & \multicolumn{2}{|c|}{ Other } \\
\hline & $\mathbf{n}$ & $\%$ & $\mathbf{n}$ & $\%$ & $\mathbf{n}$ & $\%$ & $\mathbf{n}$ & $\%$ & $\mathbf{n}$ & $\%$ & $\mathbf{n}$ & $\%$ \\
\hline Group A & 97 & 64,23 & 54 & 35,76 & 70 & 46 & 28 & 19 & 18 & 12 & 35 & 23 \\
\hline
\end{tabular}

The results for group B were moderate in $33.2 \%$, good in $13.7 \%$ and complete in $25.4 \%$ and the treatment was successful in $72.3 \%$ and unsuccessful in $27.7 \%$. After 6 months, the respective percentages for moderate, good and complete improvement were $17.2 \%, 41.1 \%$, and $17.2 \%$ with $75.5 \%$ successful and $24.5 \%$ unsuccessful in group A. The six-month figures for group B were $28.3 \%$ moderate, $1 \%$ good, and $25.4 \%$ complete improvement with the treatment being successful in $54.7 \%$ and unsuccessful in $45.3 \%$. We see that treatment of group A was more successful than group B in the 3rd month $(\mathrm{p}<0.05)$, and much more successful than group $B$ in the 6th month $(\mathrm{p}<0.001)$ in terms of the number of patients who were successfully and unsuccessfully treated according to the Quick DASH scores. These results show that a better result was obtained in group A than group B in both periods.

No significant side effect was found in group B but there was subcutaneous fat necrosis in 5 patients. These patients underwent surgical procedures when their symptoms did not decrease and they had moderate improvement postoperatively.

\section{Discussion}

Conservative methods are first used in the treatment of acute lateral epicondylitis (Papa et al., 2012). One of the most widely used methods is corticosteroid and local anesthetic injection (Altay et al., 2002; Saccomanni et al., 2010).Injection treatment is preferred because good results can be obtained in a short time. Adding physical therapy methods (stretching and lengthening exercises for the elbow extensor muscles and tendons, cold or hot compress applications, etc.) and NSAI treatments has been shown to increase the success rate and duration in many randomized studies (Smidt et al., 2002; Altay et al., 2002; Baskurt et

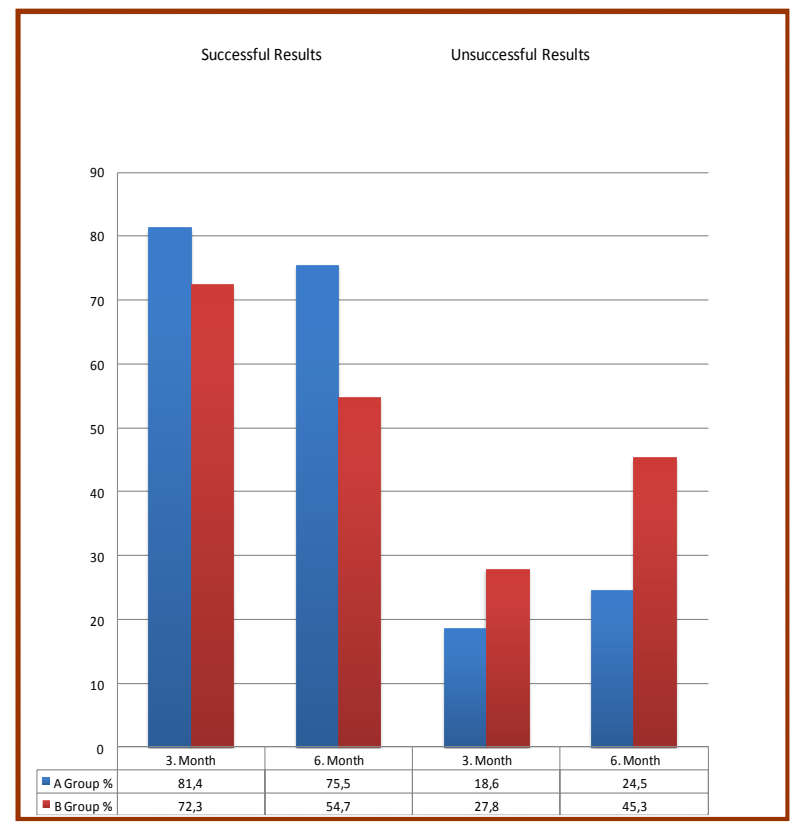

Graphic 1 : Quick DASH scores according to the groups

al., 2003; Trudel et al., 2004; Lopes et al., 2006; Bisset et al., 2006; Yarrobino et al., 2006; Papa et al., 2007; Allan et al., 2007). A study reported that $90 \%$ of lateral epicondylitis cases recovered within 6 months while the remaining $10 \%$ consisted of resistant cases and had to undergo surgery (Trinh et al., 2004). The total rate of moderate, good and very good results was $81.5 \%$ in group A and $72.3 \%$ in group B at the 3rd month and $75.5 \%$ in group A and $54.7 \%$ in group B at the 6th month. We therefore only obtained good results similar to those reported in group A. A surgical procedure was performed in $6(3.9 \%)$ patients in group A and $18(8.7 \%)$ patients in group B when conservative treatment was unsuccessful. 
Comparing steroid and local anesthetic injection treatment with placebo, local anesthetic injection, and the wait-and-see technique revealed that quite good results were obtained in the term period but no difference was present over time (Smidt et al., 2002). The effect magnitude and duration of the epicondylitis band was better than with injection treatment, both in the short term and the long term in our study.

The epicondylitis band is used commonly worldwide and has been shown to have a large contribution in resting the affected sensitive radial region, decreasing edema, and accelerating the treatment based on the force distribution principle in localizations closer to the regions where the extensor tendon originates from, although the mechanism of action is not fully clear (Struijs et al., 2004; Altan et al., 2008). It has also been demonstrated in recent years that pain decreases significantly and the capacity of movement increases in the elbow with the wrist extension orthosis used for the conservative treatment of lateral epicondylitis (Garg et al., 2010).

The rate and maintenance of successful treatment in the two groups did not very greatly with our principle of combined treatment.

Injection treatment in group B caused increased pain and movement limitation in some of our patients but these decrease after a few days. We also observed subcutaneous fat necrosis in 5 (2.4\%) patients and the number of patients requiring a 2 nd injection was $54(26.3 \%)$. There were no other complications. The epicondylitis band did not cause any complications in any of our patients. Epicondylitis band application caused difficulty in daily activities from time to time but this did not lead to disruption of the ongoing treatment. Use of the band was continued in cases with moderate scores during the next 6 months in Group A and this was seen to have positive effects on the result.

It was noteworthy that a large proportion of our patients consisted of women and most were housewives. The fact that only $7(2 \%)$ patients of our subjects played active tennis indicates that the "tennis elbow" term should be reevaluated. It is also known that lateral epicondylitis is not mostly seen in tennis players (Kaminsky et al., 2003).

We recommend that a 3rd group should be studied using a wait-and-see method, placebo, wrist extension orthosis or just steroid injections and evaluated with longer follow-up durations as the next stage of this study.

\section{Conclusion}

In conclusion, elbow pain is the main symptom in acute lateral epicondylitis and movement limitation associated with this pain is present. The first treatments that should be considered are the conservative methods of epicondylitis bandage or injection of corticosteroids and local anesthetics. Although injection treatment is particularly striking with its short-term effect, we believe that its lack of superiority regarding long-term results and the potential complications require its consideration only as a secondary plan. We also believe that an epicondylitis band with combined treatment should be considered first due to the ease of use, its lack of invasiveness or complications, and the better results with combined treatment in the long term compared to injection treatment.

Informed Consent: Verbal informed consent was obtained from patients who participated in this study.

Peer-review: Externally peer-reviewed.

Author Contributions: Concept-EA, Design-EA, TDA, Supervision- E A, Funding- EA, TDA, Materials- EA, TDA, UT, Data Collection and/or Processing- BS, Analysis and/or InterpretationEA, TDA, UT, Literature- EA, TDA, Review-EA, TDA, Writing-- EA, TDA, Critical Review-EA

Conflict of Interest: No conflict of interest was declared by the authors.

Financial Disclosure: The authors declared that this study hasn't received any financial support.

\section{References}

Allan W, Erak S, Fractional lengthening of forearm extensors for resistant lateral epicondylitis. ANZ J Surg 2007; 77: 981-4.

Altan L, Kanat E. Conservative treatment of lateral epicondylitis: comparison of two different orthotic devices. Clin Rheumatol 2008; 27: 1015-9.

Altay T, Günal I, Öztürk H. Local injection treatment for lateral epicondylitis. Clin Orthop Relat Res 2002; 398: 127-30.

Başkurt F, Özcan A, Algun C. Comparison of effects of phonophoresis and iontophoresis of naproxen in the treatment of lateral epicondylitis. Clin Rehabil 2003; 17: 96-100. 
Bisset L. Mobilisation with movement and exercise, corticosteroid injection, or wait and see for tennis elbow: randomised trial. BMJ. 2006; 333:939.

Chesterton S L, Van der Windt D, Sim J, Lewis M, Mallen C, Mason E et al. Transcutaneous electrical nerve stimulation for the management of tennis elbow: a pragmatic randomized controlled trial: the TATE trial. BMC Musculo skeletal Disorders 2009; 10:156.

Ciccotti MC, Schwartz MA, Ciccotti MG. Diagnosis and treatment of medial epicondylitis of the elbow. Clin Sports Med 2004; 23(4): 693-705.

Cohen MS, Romeo AA, Hennigan SP, Gordon M. Lateral epicondylitis: anatomic relationship of the extensor tendon origins and implications for arthroscopic treatment. J Shoulder Elbow Surg 2008; 17: 954-60.

Erak S, Day R, Wang A. The role of supinator in the pathogenesis of chronic lateral elbow pain:biomechanical study. J Hand Surg 2004; 29 b: 461-4.

Franchignoni F, Giordano A, Sartorio F, Vercelli S, Pascariello B, Ferriero G. Suggestions for refinement of the Disabilities of the Arm, Shoulder and Hand Outcome Measure (DASH): a factor analysis and Rasch validation study. Arch Phys Med Rehabil 2010; 91: 1370-7.

Garg R, Adamson GJ, Dawson PA, Shankwiler JA, Pink MM. A prospective randomized study comparing a forearm strap brace versus a wrist splint for the treatment of lateral epicondylitis. J Shoulder Elbow Surg 2010: 19: 508-12.

Jobe FW, Ciccotti MG. Lateral and medial epicondylitis of the elbow. J Am Acad Orthop Surg 1994; 2:1-8.

Johns LD. Non thermal effect soft herapeutic ultrasound: the frequency resonance hypothesis. J Athl Train 2002; 37: 293-9.

Kaminsky S, Baker C. Lateral epicondylitis of the elbow. Tech Hand Upper Surg 2003; 7: 179-89.

Lopes-Martins RA, Albertini R. Steroid receptor antagonist mifepristone inhibits the anti inflammatory effects of photoradiation. Photomed Laser Surg 2006; 24(2): 197-201.

Meyer NJ, Pennington W, Haines B. The effect of the forearm support band and forces at the origin of the extensor carpi radialisbrevis: A cadaveric study and review of literature. J Hand Ther 2002; 15(2): 179-84.

Nirschl RP, Pettrone FA. Tennis elbow: the surgical treatment of epicondylitis. J Bone Joint Surg Am 1979; 61: 832-9.
Oksüz Cigdem, Quick DASH-Turkish by Çiğdem Öksüz MS, Tülin Düger Assoc. Prof Hacettepe University School of Physical Therapy and Rehabilitation www.drsedatyildiz.com/FileUpload/ks109487/ File/quickdash turkey.pdf. 2006

Papa JA. Two cases of work-related lateral epicondylopathy treated with Graston Technique ${ }^{\circledR}$ and conservative rehabilitation $\mathrm{J}$ Can Chiropr Assoc 2012; 5(3): 192-200.

Pomerance J. Radiographic analysis of lateral epicondylitis. J Shoulder Elbow Surg 2002; 11: 156-7.

Saccomanni B. Corticosteroid injection for tennis elbow or lateral epicondylitis: a review of the literature. Curr Rev Musculoskelet Med 2010; 3:38-40.

Smidt N, Assendelft WJ. Corticosteroid injections for lateral epicondylitis: a systematic review. Pain 2002; 96: 23-40.

Smidt N, Van der Windt DA, Assendelft WJ, Devillé WL, Korthals-de Bos IB, Bouter LM. Corticosteroid injections, physiotherapy, or a wait-and-see policy for lateral epicondylitis: a randomised controlled trial. The Lancet 2002; 359: 657-62.

Struijs PA, Kerkhoffs GM, Assendelft WJ, Van Dijk CN. Conservative treatment of lateral epicondylitis brace versus physical therapy or a combination of both a randomized clinical trial. Am J Sports Med.2004; 32: 462-9.

Trinh KV, Phillips SD, Ho E, Damsma K. Acupuncture for the alleviation of lateral epicondyle pain: a systematic review. Rheumatology 2004; 43: 1085-90.

Trudel D, Duley J, Zastrow I, Kerr EW. Rehabilitation for patients with lateral epicondylitis: a systematic review. J Hand Ther 2004; 17: 243-66.

Uzunca K, Birtane M, Tastekin N. Effectiveness of pulsed electromagnetic field therapy in lateral epicondylitis, Clin Rheumatol. 2007; 26(1): 6974.

Yarrobino E T, Kalbfleisch H J, Panus C P. Lidocainei onto phoresis mediates analgesia in lateral epicondylalgia treatment. Physiother Res Int. 2006; 11: 152-60. 